\title{
Comparison of Ondansetron versus Acupressure on Pressure Point Neiguan (P-6) to Prevent Post Operative Nausea and Vomiting after Middle Ear Surgery: A Randomized Double Blind Study
}

\author{
Authors \\ *Sanjeev Kumar ${ }^{1}$, Chandrakant Prasad ${ }^{2}$, Rajnish Kumar $^{3}$, Kishore $^{4}$, Ravi Anand $^{5}$, \\ Rakesh Kumar Singh ${ }^{6}$, K. H. Raghwendra ${ }^{7}$ \\ ${ }^{1}$ Addl. Professor, Dept. of Anaesthesiology \& Critical Care Medicine, IGIMS, Patna, \\ ${ }^{2}$ Junior Resident, Dept. of Anaesthesiology \& Critical Care Medicine, IGIMS, Patna \\ ${ }^{3}$ Associate Professor, Dept. of Anaesthesiology \& Critical Care Medicine, IGIMS, Patna \\ ${ }^{4}$ Ex. Senior Resident, Dept. of Anaesthesiology \& Critical Care Medicine, IGIMS, Patna \\ ${ }^{5}$ Ex. Junior Resident, Dept. of Anaesthesiology \& Critical Care Medicine, IGIMS, Patna \\ ${ }^{6}$ Professor \& Head, Dept. of Advanced ENT, IGIMS, Patna \\ ${ }^{7}$ Professor, Dept. of Anaesthesiology \& Critical Care Medicine, IGIMS, Patna \\ *Corresponding Author \\ Dr Sanjeev Kumar, MBBS, MD (BHU, Varanasi) \\ Addl. Professor, Dept. of Anaesthesiology \& Critical Care Medicine, IGIMS, Patna, India \\ Email: sanjeev_pranay71@hotmail.com
}

\begin{abstract}
Aim: The aim of this study was to compare the efficacy of intravenous ondansetron with P6 acupuncture stimulation in prevention of post-operative nausea and vomiting after middle ear surgery.

Materials and Methods: After obtaining institutional research and ethical committee approval. 90 Patients of ASA physical status I \& II, aged 20 to 60 years, posted for middle ear surgery under general anaesthesia were randomly allocated into two groups by computer generated random number method into Group A (n=45) - acupressure on Pressure point P-6 \& Group B $(n=45)$ ondansetron $0.1 / \mathrm{kg}$ after endotracheal intubation. Balanced general anaesthesia was given as per preset protocol and intraoperative vitals were recorded at regular intervals. The incidence of nausea and vomiting was recorded every 6 hours for a period of 24 hours by direct questioning to the patient or to his attendant. Nause and vomiting were evaluated on a three point scale.

Results: There was no significant difference in number of patients having nausea or vomiting at any interval in both the groups i.e., p-value $>0.05$ at all intervals.

Conclusion: We conclude that P6 acupuncture stimulation is almost equally effective as to intravenous ondansetron in prevention of post-operative nausea and vomiting after middle ear surgery.

Keywords: P-6 pressure point, acupuncture, ondansetron, middle ear surgery, post-operative, nausea and vomiting.
\end{abstract}




\section{Introduction}

Post-operative nausea vomiting is a leading complication of middle ear surgery and is often viewed by patients as a single most common side effect of anaesthesia and operative period. Without any prophylaxis, PONV occurs in approximately $20-30 \%$ of the general surgical population and up to $70-80 \%$ in patients with predisposing risk factors. As anaesthetic duration increases, risk of PONV also increases. When the risk is sufficiently great, prophylactic antiemetic medications are administered and strategies to reduce its incidence are initiated. Incidence of PONV varies according to several variables, including the patient age, weight, sex, high risk surgeries such as gynaecological surgeries, middle ear surgeries and laparoscopic surgeries etc. Postoperative nausea and vomiting (PONV) is not only an unpleasant symptom for patients ${ }^{1}$, but can also delay hospital discharge and increase use of resources $^{2}$. The overall incidence of PONV is approximately $30 \%^{3}$, increasing up to $79 \%$ in high risk patients ${ }^{4}$.

There are 8 classes of antiemetic agents that are typically used for PONV includes phenothiazines, anticholinergics, anti-histamines, butyrophenones, substituted benzamides, corticosteroids, 5- $\mathrm{HT}_{3}$ receptor antagonists, and $\mathrm{NK}_{1}$ receptor antagonists. The side effect associated with these drugs includes headache, constipation, agitation, tachycardia, extra pyramidal effect, sedation and even possibly prolonged QT interval and fatal arrhythmias.

Ondansetron is a carbazolone derivative that is structurally related to serotonin and possesses specific 5-HT3 subtype receptor antagonist properties, without altering dopamine, histamine, adrenergic, or cholinergic receptor activity. Cardiac dysrhythmias and conduction disturbances (atrioventricular heart block) have been reported after the intravenous administration of ondansetron. Ondansetron and other 5-HT3 receptor antagonists can cause slight prolongation of the QTc interval on the electrocardiogram of treated patients, but this has not created the same level of concern as that ascribed to droperidol.
Ondansetron, 4 to $8 \mathrm{mg}$ IV (administered over 2 to 5 minutes immediately before the induction of anaesthesia) is highly effective in decreasing the incidence of postoperative nausea and vomiting in a susceptible patient population.

Acupuncture is a complementary technique originated in China in which various diseases and disorders can be treated by the application of fine needle punctures at strategic points called acupuncture points or acupoints in the body. P6 is an acupuncture point in the body located 4 to $5 \mathrm{~cm}$ proximal to distal wrist crease between the tendons of flexor carpiradialis and Palmarislongus (figure 1). There are several studies which have shown a significant role of P6 acupoint stimulation in prevention of postoperative nausea and vomiting.

Our objective in conducting this study was to compare the efficacy of intravenous ondansetron with P6 acupuncture stimulation in prevention of post-operative nausea and vomiting after middle ear surgery.

\section{Figure 1}

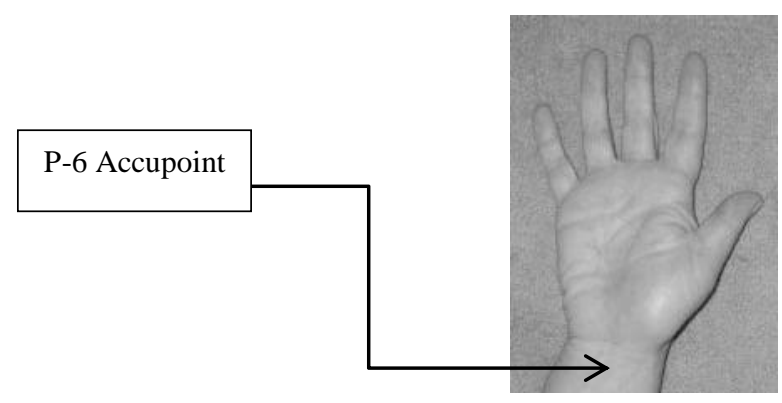

\section{Materials and Methods}

After obtaining institutional research and ethical committee approval, this prospective randomized double blind comparative clinical study was conducted at Indira Gandhi Institute of Medical Sciences, Patna.90 Patients of ASA physical status I $\&$ II, aged 20 to 60 years, posted for middle ear surgery under general anaesthesia were enrolled in this study. Patients with known allergy to ondansetron, refusing for participation, having pregnancy or GERD or having history of previous PONV\& mental retardation were excluded from the study. Patients were randomly allocated into two 
groups by computer generated random number method into two groups

Group-A ( $\mathrm{n}=45)$ : Acupressure on pressure point P-6 Group-B $\quad(n=45)$ : Ondansetron $\quad 0.1 / \mathrm{kg}$ after endotracheal intubation

6 hours fasting status was maintained in all the patients for solid food and clear fluids was allowed until 2 hours preoperatively. A spherical bead of acupressure wristbands was placed at the P6 points on both forearms in the acupressure group (Group A) patients. In the control group (Group B), the spherical bead of acupressure wristbands was placed on the posterior surface. The acupressure wristbands were applied $30 \mathrm{~min}$ before induction of anaesthesia. The treatment point P-6 (Nei-Guan) was located on the anterior surface of the forearm, approximately $1 \mathrm{~cm}$ deep to the skin, two body inches proximal to the distal crease of the wrist joint between the tendons of flexor-carpiradialis and palmarislongus. One body inch is equal to the width of the interphalangeal joint of patient's thumb.

The patients of group-A wore bilateral elastic acupressure wristbands applying pressure on the $\mathrm{p}$ 6 acupressure points while that of Group B received placebo wristbands consisting of bands without pressure buttons. Both groups were applied the bands at least 30 minutes prior to induction of general anesthesia by an anesthesia provider who was not involved in data collection. Bands were loosely covered with gauze and tape so that the groups were indistinguishable from each other thus blinding the data recorders, subjects and anaesthesia providers to the treatment group. The provider applying the bands would check A or B on the protocol checklist. Subjects were instructed to leave the bands in place for 24 hours following surgery unless discomfort prevented them from doing so.

On the day before the surgery all the patients were clinically evaluated, assessed and investigated. The study protocol was explained to the patient and written informed consent was taken from each participant. All patients received oral pantoprazole $40 \mathrm{mg}$ as pre-anaesthetic medication at 6AM on the day of surgery.
In Operation Theater after securing intravenous line, standard monitoring includes (ECG, NIBP and pulse oximetry) were attached and base line parameters were recorded. General anaesthesia was given with intravenous fentanyl $2 \mu \mathrm{g} / \mathrm{kg}$, Propofol 2 $\mathrm{mg} / \mathrm{kg}$ followed by atracurium $0.5 \mathrm{mg} / \mathrm{kg}$ to facilitate insertion of endotracheal tube of appropriate size. Anaesthesia was maintained with isoflurane in a mixture of oxygen and nitrous oxide. Intra operative muscle relaxation was achieved with intermittent atracurium as required. Ventilation was mechanically controlled and adjusted to maintain $\mathrm{ETCO}_{2}$ at $32-40 \mathrm{mmHg}$ with an Anaesthetic / Respiratory analyzer (Drager Fabius GS). Intraoperative monitoring included ECG, pulse oximetry, $\mathrm{ETCO}_{2}$, systolic, diastolic and mean blood pressure, which were recorded every 5 minutes. Boluses of injection Fentanyl $1 \mathrm{mcg} / \mathrm{kg}$ intravenously was given if the heart rate and blood pressure increased more than $30 \%$ of the preoperative baseline. Injection paracetamol $15 \mathrm{mg} / \mathrm{kg}$ was given as intravenous infusion unless contraindicated. After completion of surgery, neuromuscular blockade was reversed with neostigmine $0.04 \mathrm{mg} / \mathrm{kg}$ and glycopyrrolate $0.01 \mathrm{mg} / \mathrm{kg}$ and tracheal extubationwas done after adequate reversal of muscle relaxant. Post operative analgesia was maintained by intravenous diclofenac $75 \mathrm{mg}$ every twelve hours and paracetamol $1 \mathrm{gm}$ eight hourly.

The incidence of nausea and vomiting was recorded every 6 hours for a period of 24 hours by direct questioning to the patient or to his attendant by the same anaesthesiologist No distinction was made between vomiting and retching (retching event was considered as vomiting event). Nausea and vomiting were evaluated on a three point scale $0=$ none

$1=$ nausea

2=vomiting

Rescue antiemetic medication, if required, were given in the form of Ondansetron $4 \mathrm{mg}$, repeated, if the patient experienced severe nausea, if there were more than 3 emetic episodes within a period of 15 minutes or if the patient asked for it. Adverse effects if any were recorded in all patients. 


\section{JMSCR VoI||05||Issue||07||Page 25025-25030||July}

\section{Statistical analysis}

Data were entered into Microsoft Excel spread sheet. Sample size was calculated using Lambdawillis formula based on data of previous studies. With power of study $80 \%$ and alpha error $5 \%$, the sample size came to 42 for each group. Considering drop outs, 45 patients were recruited in each group. SPSS for Windows 21 (SPSS, Chicago, IL, USA) was used for statistical analysis. Continuous variables were analyzed with the unpaired t test and categorical variables were analyzed with the ChiSquare Test and Fisher Exact Test. Statistical significance was taken as $\mathrm{P}<0.05$.

\section{Results}

There were no dropouts so all 90 patients were analyzed for results.

The demographic profiles of both the groups were comparable as shown in Table 1 \& Figure 1.

Table 1: demographic profile of both the groups

\begin{tabular}{|c|c|c|c|c|}
\hline Variable & $\begin{array}{c}\text { Group } \\
\text { A }\end{array}$ & $\begin{array}{c}\text { Group } \\
\text { B }\end{array}$ & $\begin{array}{c}\text { p- } \\
\text { value }\end{array}$ & Rem \\
\hline Age & $\begin{array}{c}31.2 \pm \\
4.32\end{array}$ & $\begin{array}{c}32.1 \pm \\
4.21\end{array}$ & 0.625 & $\begin{array}{c}\text { Non- } \\
\text { significant }\end{array}$ \\
\hline Weight (kgs) & $\begin{array}{c}61.49 \pm \\
3.35\end{array}$ & $\begin{array}{l}59.35 \\
\pm 3.61\end{array}$ & .355 & $\begin{array}{c}\text { Non- } \\
\text { significant }\end{array}$ \\
\hline Heig & $\begin{array}{c}166.21 \pm \\
5.35\end{array}$ & $\begin{array}{l}161.35 \\
\pm 7.61\end{array}$ & 0.521 & $\begin{array}{c}\text { Non- } \\
\text { significant }\end{array}$ \\
\hline Sex ratio (M:F) & $28: 17$ & $25: 20$ & 0.232 & $\begin{array}{c}\text { Non- } \\
\text { significant }\end{array}$ \\
\hline $\begin{array}{l}\text { Duration of } \\
\text { surgery (mins) }\end{array}$ & $\begin{array}{c}78.55 \pm \\
7.82\end{array}$ & $\begin{array}{c}81.47 \pm \\
9.44\end{array}$ & 0.424 & $\begin{array}{c}\text { Non- } \\
\text { significant }\end{array}$ \\
\hline
\end{tabular}

Figure 1: Demographic profile of both the groups

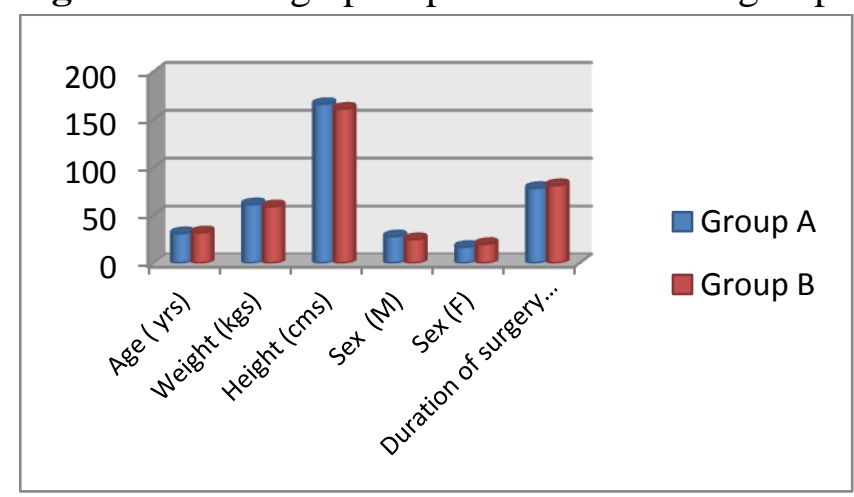

Intra-operative hemodynamic parameters were almost stable and comparable in both the groups as in Figure $2 \& 3$.
Figure 2: Intra-operative average MAP of both the groups

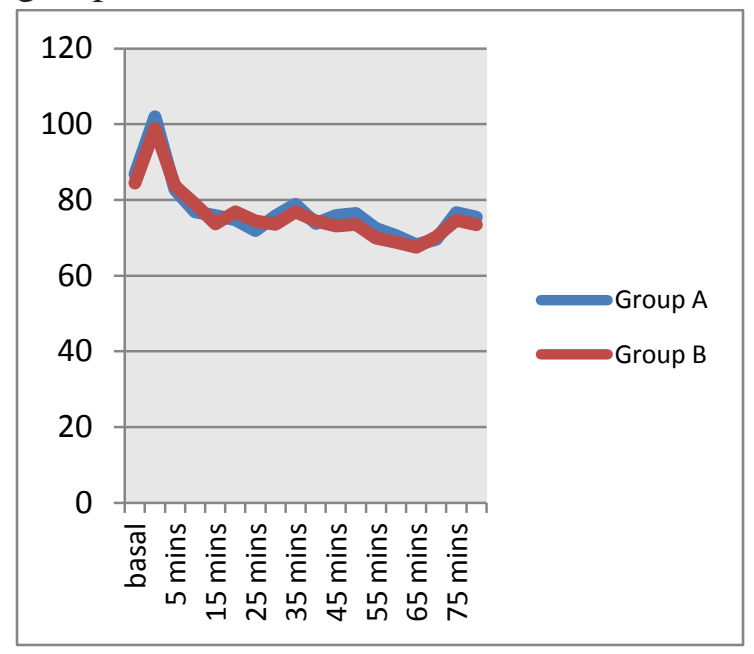

Figure 3: Intra-operative average HR of both the groups

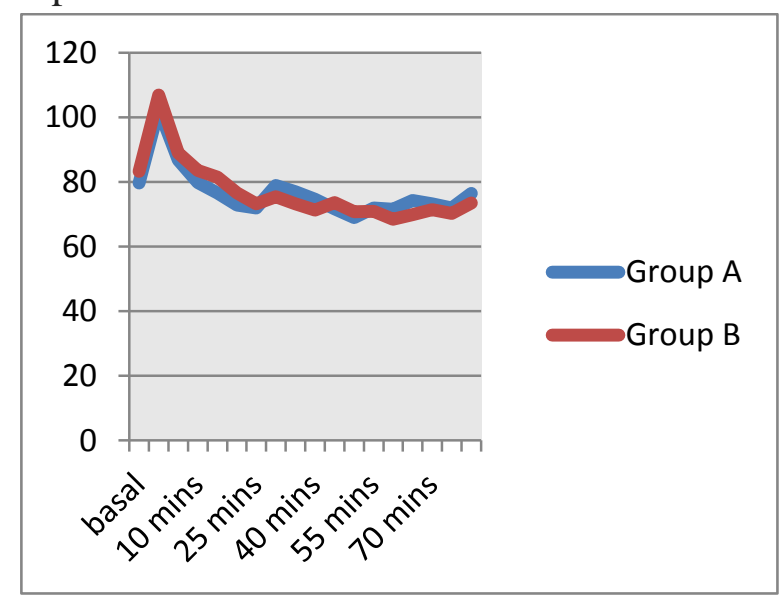

Number of patients having nausea or vomiting at 6 hours, 12 hours, 18 hours and 24 hours interval post-operatively in both the groups is shown in Table 2 and Figure 4. There was no significant difference in number of patients having nausea or vomiting at any interval in both the groups i.e, pvalue $>0.05$ at all intervals.

Table 2: Number of patients having nausea or vomiting at different interval in both the groups

\begin{tabular}{|l|c|c|c|c|}
\hline & \multicolumn{2}{|c|}{ Group A } & \multicolumn{2}{c|}{ Group B } \\
\hline & Nausea & Vomiting & Nausea & Vomiting \\
\hline 6 hours & 4 & 2 & 3 & 2 \\
\hline 12 hours & 5 & 2 & 6 & 2 \\
\hline 18 hours & 7 & 1 & 6 & 0 \\
\hline 24 hours & 4 & 0 & 3 & 0 \\
\hline
\end{tabular}

$\mathrm{P}$-value> 0.05 at all intervals 
Figure 4: Number of patients having nausea or vomiting at different interval in both the groups

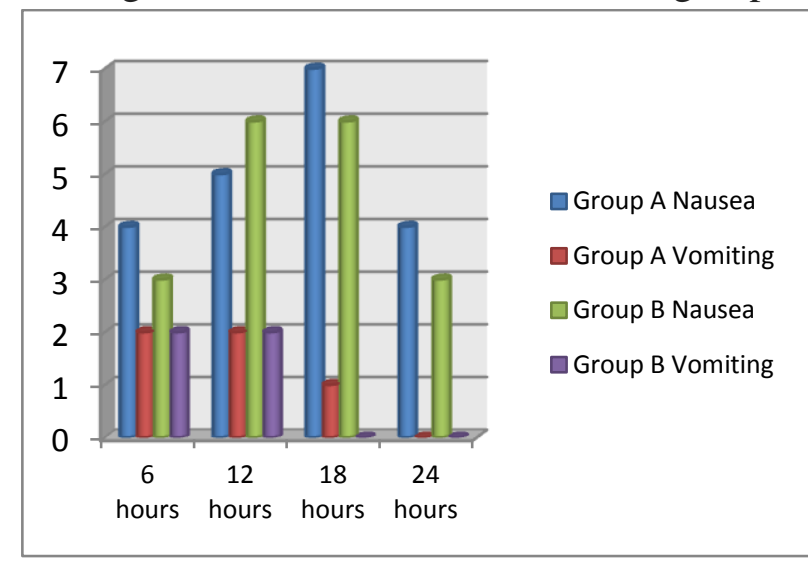

\section{Discussion}

Middle ear surgeries are invariably associated with post-operative nausea and vomiting. Many pharmacological and non-pharmacological measures have tried to alleviate this complication. Here in this study we compared the efficacy of intravenous ondansetron with P6 acupuncture stimulation in prevention of post-operative nausea and vomiting after middle ear surgery and we found P6 acupuncture is almost equally effective in this regard to intravenous ondansetron.

Acupressure is one of the non-pharmacological methods which can prevent PONV. Several studies have shown that it was an effective method which could be used for the management of PONV $^{5,6}$. The mechanism of action of Acupressure is still unclear. Several hypotheses have been described this as ambiguity, but it might be due to its influence on restoration of the body's energy balance ${ }^{7}$. In Chinese medicine, P6 point is called as the peak of body energy ${ }^{8}$ and therefore, when Acupressure is competently applied, the body's energy balance will be restored. In other studies, the effect of Acupressure at P6 was confirmed in various medical interventions ${ }^{9,10}$. However, these results were in contrast with those of other studies ${ }^{11-13}$. The some additional theory behind this is the physical part of their body always regulated by metaphysical part. Metaphysical energy is always performing through metaphysical activity and stimulation. This type of stimulation is generally perform while walking, massage of the body and barefoot walking and also though the proper massage of relevant points and meridians. The energy field of the target organ when properly balanced by the acupressure methods controls the negative symptoms (PONV) of the body.

Acupuncture and acupressure can provide relief but due to provider proficiency, patient acceptance and proven efficacy compared to antiemetic medications, these methods are less frequently used $^{14}$.Lee et $\mathrm{al}^{14}$ recommended acupressure wristbands may be effective in preventing PONV after short surgical procedures when applied prior to emetic stimulus exposure such as anaesthetic agents (PONV incidence 23\% in treatment group, $41 \%$ in placebo group; $\mathrm{p}=0.0058$ ). White et $\mathrm{al}^{15}$ compared acupuncture with ondansetron and found that both therapies were similar for preventing PONV. However, the combination of acupuncture and ondansetron was better than ondansetron alone in preventing nausea and vomiting (20\% Vs 50\% for nausea; $\mathrm{p}<0.05$ and $0 \%$ Vs $20 \%$ for vomiting; $\mathrm{p}<0.05$, respectively). When compared with placebo, Korean acupressure when used points on the fingers rather than the wrists, significantly reduced nausea and vomiting $(40 \%$ Vs. $70 \%, \mathrm{p}=$ $0.006 ; 22.5 \%$ Vs $50 \%, \mathrm{p}=0.007$, respectively). ${ }^{16}$

\section{Conclusion}

We found in this study that P6 acupuncture stimulation is almost equally effective as to intravenous ondansetron in prevention of postoperative nausea and vomiting after middle ear surgery.

\section{References}

1. Macario A, Weinger M, Carney S, Kim A. Which clinical anesthesia outcomes are important to avoid? Anesthesia and Analgesia 1999; 89: 652-8.

2. Hill RP, Lubarsky DA, Phillips-Bute B, et al. Cost-effectiveness of prophylactic antiemetic therapy with ondansetron, droperidol, or placebo. Anesthesiology 2000; 92: 958-67. 
3. Watcha MF, White PF. Postoperative Nausea and Vomiting. Anesthesiology 1992; 77: 16284.

4. Apfel CC, Greim CA, Haubitz I, et al. A simplified risk scores for predicting postoperative nausea and vomiting: Conclusions from cross-validations between two centers. Anesthesiology 1999; 91: 693700 .

5. EbrahimSoltani AR, Mohammadinasab $\mathrm{H}$, Goudarzi M, Arbabi S, Mohammadinasab A, Mohammadinasab F, et al. Comparing the efficacy of prophylactic p6 acupressure, ondansetron, metoclopramide and placebo in the prevention of vomiting and nausea after strabismus surgery. Acta Med Iran. 2011; 49(4):208-12.

6. McCracken G, Houston P, Lefebvre G. Guideline for the management of postoperative nausea and vomiting. J Obstet Gynaecol Can. 2008 Jul; 30(7): 600-7, 8-16.

7. Direkvand-Moghadam A, Rezaeian $\mathrm{M}$. Increased intravenous hydration of nulliparas in labor. Int J Gynaecol Obstet. 2012 Sep; 118(3):213-5.

8. Wallenborn J, Gelbrich G, Bulst D, Behrends $\mathrm{K}$, Wallenborn $\mathrm{H}$, Rohrbach $\mathrm{A}$, et al. Prevention of postoperative nausea and vomiting by metoclopramide combined with dexamethasone: randomised double blind multicentre trial. BMJ. 2006 Aug 12; 333(7563):324.

9. Gan TJ, Jiao KR, Zenn M, Georgiade G. A randomized controlled comparison of electroacupoint stimulation or ondansetron versus placebo for the prevention of postoperative nausea and vomiting. AnesthAnalg. 2004 Oct; 99(4):1070-5.

10. Coloma M, White PF, Ogunnaike BO, Markowitz SD, Brown PM, Lee AQ, et al. Comparison of acustimulation and ondansetron for the treatment of established postoperative nausea and vomiting. Anesthesiology. 2002 Dec; 97(6):1387-92.

11. Genc A, Can G, Aydiner A. The efficiency of the acupressure in prevention of the chemotherapy-induced nausea and vomiting. Support Care Cancer. 2013 Jun 9; 21(1):25361.

12. Lee A, Fan LT. Stimulation of the wrist acupuncture point P6 for preventing postoperative nausea and vomiting. Cochrane Database Syst Rev. 2009(2): CD003281.

13. Fan C, Tanhui E, Joshi S, et al. Acupressure treatment for prevention of postoperative nausea and vomiting. Anesth Analg 1997; 84:821-5.

14. Lee A, Done ML: The use of nonpharmacologic techniques to prevent postoperative nausea and vomiting: A metaanalysis. AnesthAnalg 1999; 88: 1362

15. White PF, Issioui $\mathrm{T}, \mathrm{Hu} \mathrm{J}$, et al. Comparative efficacy of acustimulation (Relief Band) versus ondansetron (Zofran) in combination with droperidol for preventing nausea and vomiting. Anesthesiology 2002; 97:1075-81.

16. Boehler M, Mitterschiffthaler G, Schlager A. Korean hand acupressure reduces postoperative nausea and vomiting after gynecological laparoscopic surgery. AnesthAnalg 2002; 94:872-5. 